\title{
Some Contributions to Surgery of the Hand
}

\author{
By WILLIAM GIRDWOOD, B.Sc. (Med.), M.Ch.(Rand), F.R.C.S.(Edin.), F.R.C.S.(Eng.), Johannesburg. \\ (Reproduced by kind permission of the "S.A. Medical Journal."
}

\begin{abstract}
MAKE no apology for discussing a subject as humble as the hands; much original thought and genuine progress have come from the application of sincere study and accurate observations on the meanest problems. Moreover, because of its importance to mankind, the hand has attracted the attention of surgeons throughout the years.
\end{abstract}

Johannesburg-and the University of the Witwatersrand - has reason to be proud of its contributions to the anatomy and surgery of the hand. Four theses have been written by its sons, namely, Mr. Jack Allen, Mr. Trevor Jones, Mr. H. Gaylis and myself. Mr. Lee McGregor's Synopsis of Anatomy has further contributed to the anatomy of the hand.

Much of the work to be discussed in this paper was done 18 years ago. At that time, Kanavel and Shaw's valuable contributions on the hand had been published almost as long again before.

The character of an animal is reflected in the structure of the upper limb. The activities of civilised man and his pursuits as workman or artist are reflected in the close brain-eye-hand correlation that is man's chief characteristic. The distal articular surface of the metacarpo-phalangeal joint, in particular, is different in man as compared with apes; so, too, are the metacarpal heads. Apart from the more powerful opposable thumb of man, the main difference between the hand of man and ape are to be seen in the metacarpo-phalangeal joints. The human being has more side movement in fiexion than the limited up-and-down movement in apes, to favour thumb-index sensory and motor approximation and the formation of a fist.

It behoves surgeons to be interested in hand surgery. Today there is a vast amount of industrial W.C.A. hand surgery that required treatment according to the highest tenets of our profession. It is only through the surgical teachers that basic knowledge concerning hand surgery is handed on to our students. Hand surgery has become a casualty problem, and surgeons who practised in the 'golden era' of hand surgery, when at least $20 \%$ of hospital beds in surgical wards were occupied by hand cases, must realise that a low ebb has been reached in the teaching of hand surgery.

Hand surgery is the meeting place for many branches of nedicine. Here the general practitioner, general surgeon, orthopaedic surgeon and plastic surgeon can show a common interest; and the physician, neurologist and dermatologist are not excluded.

In this paper I shall discuss why a hand goes wrong under treatment, and how we can endeavour to put it right.

\section{THE FIXED CLAW HAND}

In this type of case (Fig. 1) there is practically no movement at the interphalangeal (IP) joints, the wrist is flexed and possibly fixed, the thumb adducted, the metacarpo-phalangeal (MP) joints extended. There is wasting, deformity and severe loss of function. The only help 18 years ago was from Kanavel, who showed in 1929 that a hand allowed to be in a bad position develops a fixed claw hand, whereas a hand in the position of function recovers. He said that in infections the invasion spreads up the lumbrical canals, round the capsule, and that this causes a fixed claw hand. Apart from infection, however, this condition is seen in

\footnotetext{
* Paper presented at the combined Surgical Forum of the Department of Surgery, University of the Witwatersrand, and the Association of of Surgery, University of the Witwatersrand, and the Association of
Surgeons of South Africa (Johannesburg Sub-group) on 8 July, 1958, and at a symposium on Hand Surgery, Rehabilitation Association,
} orenthurst Clinic, Johannesburg, on 15 October, 1958. burns where only skin is involved, and in immobilised hands.

For instance, it has been seen after a wrist dislocation, in severe crippling pain after herpes zoster, and in radial palsy, single extensor tendon injuries and other lesions not related in any way to infection.

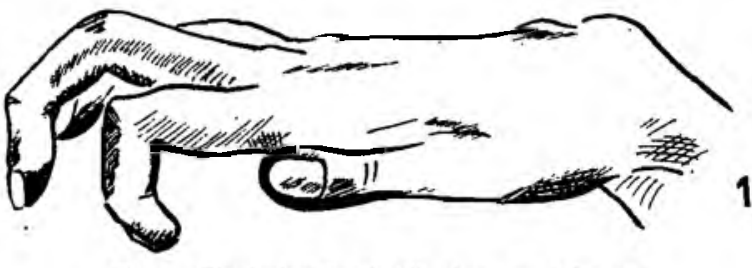

Fig. 1. Fixed claw hand following carpal fracture.

Intrinsic Muscles on Movenent Vary in Position and Function.

Studying the MP joints, let us note that the distal articular surface is an extended structure consisting not only of the distal bony articular surface but also of a thickening of the volar capsules-the palmar ligament. This receives on its sides a special portion of the collateral ligament and a portion of the insertion of the dorsal interossei. Dissections during anatomical courses do not involve study of this view of the anatomy, and much is lost thereby. (See Fig. 2, A and B).

This extended distal articular surface glides over the metacarpal head from extension to flexion and, by reason of the direct and indirect attachment to it of the dorsal and volar interossei, the line of axis of the muscles and the functional activities vary according to the particular position at the time.

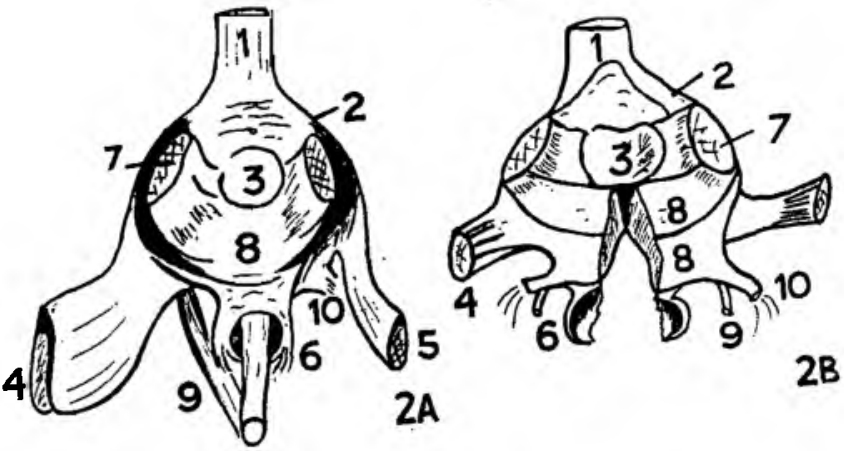

Fig. 2. (A) Right index finger, distal articular structures.

(B) Middle finger distal articular structures.

1, Extensor tendon 2, Transverse fibres of aponeurosis. 3, Proximal 1, Extensor tendon. 2, Transverse fibres of aponeurosis. 3, Proximal interosseous muscle. 6 , Fibrous connection to palmar fascia and fibrous interosseous muscle. 6, Fibrous connection to palmar fascia and fibrous
flexor sheath. 7, Collateral ligaments. 8, Portion of collateral ligament forming palmar ligament. 9, Lumbrical muscle. 10, Deep transverse palmar ligament.

In extension the dorsal interossei can abduct, but otherwise they tend to pull the articular surfaces together and jam the palmar ligamant against the metacarpal head.

The volar interossei may actually hyperextend and adduct the fingers, and this is the position usually seen in the late fixed claw hand where the dorsal interossei seem to have wasted through inactivity, and the volar interossei show a 

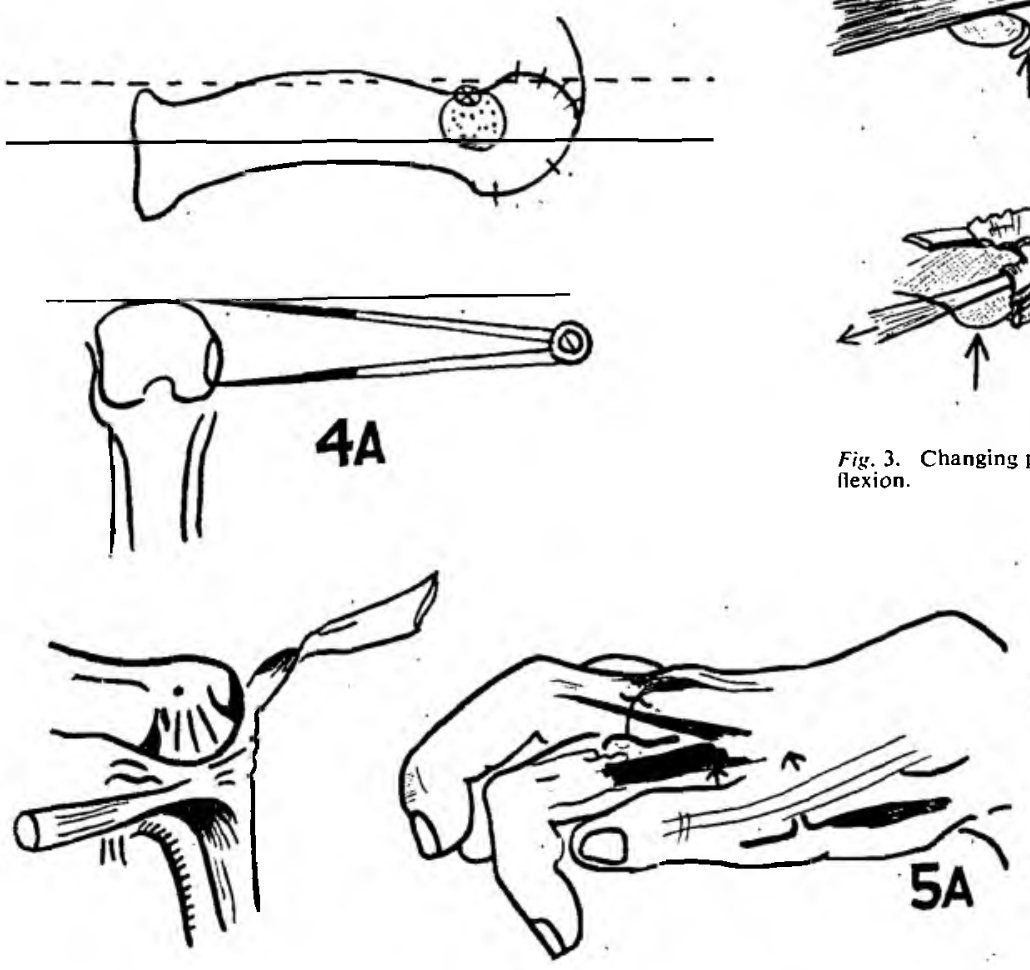

Fig. 3. Changing position of dorsal and volar interossei in extension and flexion.
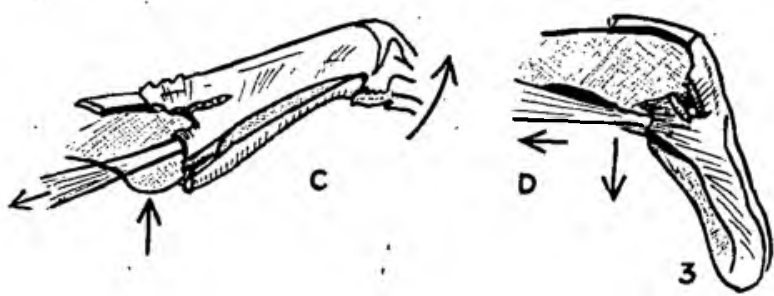

B
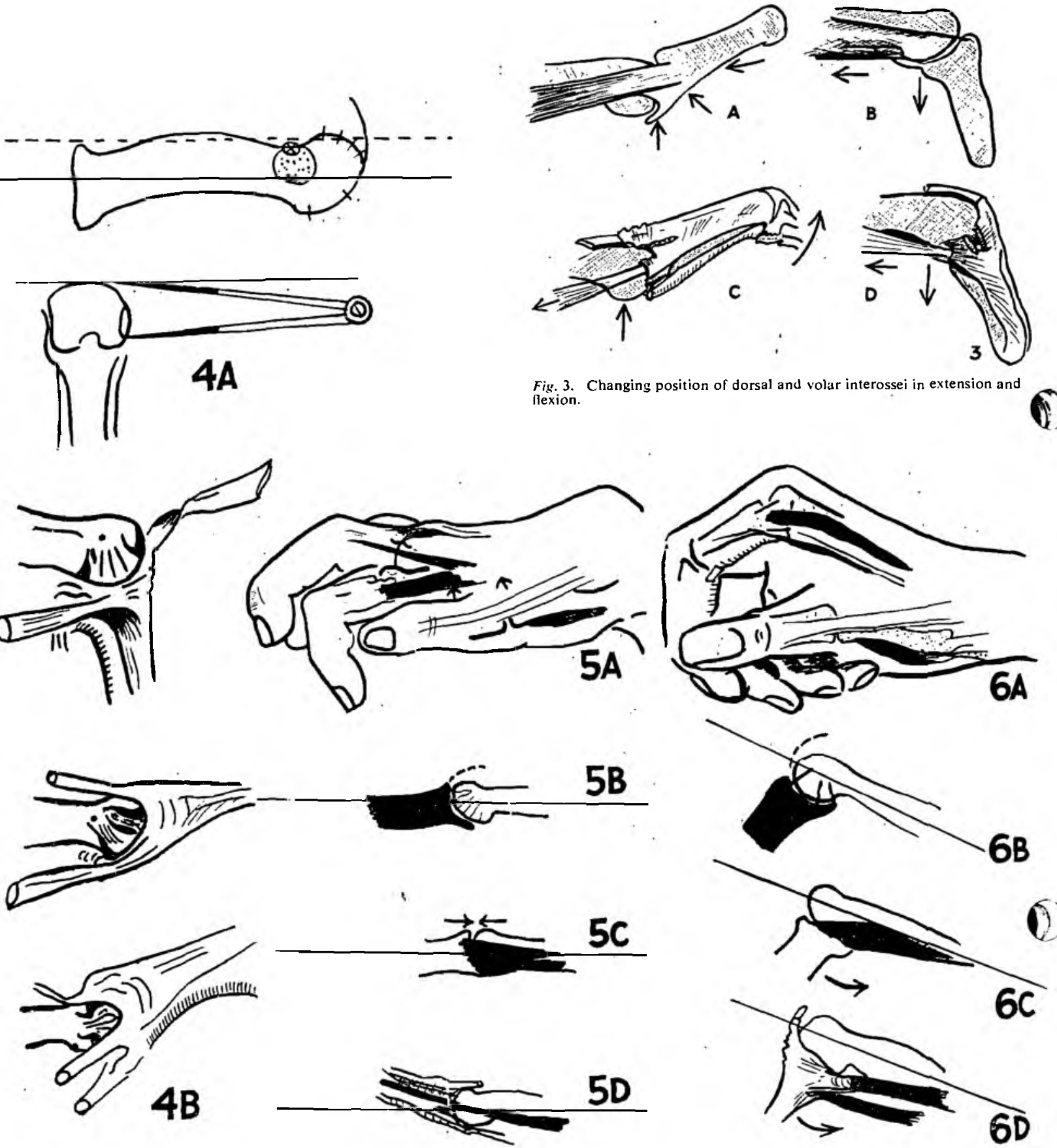

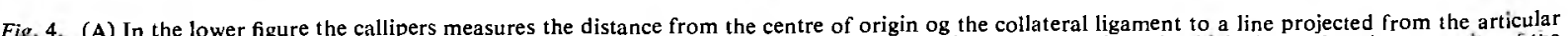

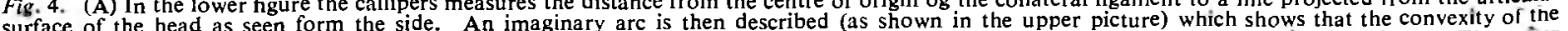

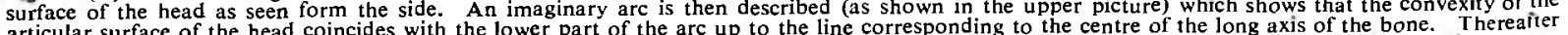
articular surface of the head coincides with the lower part of the arc up to the line corresponding to the centre of the long axis of the bone. The

(B) Collateral ligaments of MP joint in 3 positions

Fig. 5 . (A) Fixed claw hand.

(B) Relaxation of dorsal fibres of collateral ligament in extension of MP join

(C) Dorsal interosseous muscle pulling joint surfaces together in extension.

Fig. 6. (A) Hand in position of function

(B) Collateral ligament taut in flexion of MP joint.

(B) Collateral ligament taut in fiexion of MP joint.

(D) Vorsal interosseous muscle in position of mechanical advantage (in fiexion of MP joint). 
dominance over the dorsal interossei and produce the characteristic deformity.

In flexion the dorsal interossei assist flexion and lateral movements. The volar interossei will also partake in flexion of the MP joints. In extension, maximum contraction will produce minimum movement, whereas in flexion minimum contraction will permit a maximum movement and assist recovery. This concept of altering anatomy and function in different positions gives a dynamic concept to the study of the hand, with constant variations and changes in mechanics and functions (Fig. 3).

\section{Collateral Ligamants.}

When there is extension and hyperextension of the MP joints, the dorsal fibres of the collateral ligamants are relaxed, but in flexion the fibres are taut. In the IP joints, the collateral ligament is broad and the head is more circular, so that the arc of the fibres is such that with any position of the IP joint some fibres are relaxed. These joints, therefore may become stiff in any position if kept in that position long enough, whereas it is safe to keep a MP joint flexed as long as one likes. (See Fig. 4, A and B.)

In prolonged immobilization of fingers in extension, especially of the ring or little fingers, a snap will take place from extension to flexion on sustained pressure, and vice versa. This is the 'snap phenomenon', due to stretching of the collateral ligamant over the most prominent point of the metacarpal head. Microscopically, it is seen in such a case that a bunching up of the fibres of the collateral ligament has happened, and this is seen too in the nuclei. The fibres are wavy instead of straight, the nuclei also wavy and closer together-bunched up, as it were-and corresponding to the clinical state of fixation.

When a MP joint gets stiff from immobilisation in extension, the palmar ligament is jammed against the metacarpal head through the pull of the dorsal interosseous insertions, and attempts to flex the joint are ineffective and only a 'pseudo-flexion' will take place. The dorsal part of the joint may be stretched open, but there is no gliding of the distal articular surface over the head of the metacarpal into flexion.

\section{Fully Developed Fixed Claw Hand.}

Fixed claw hand is a fully developed deformity dependent on an accumulated disability associated with the effects of wrist drop, prolonged extension of the MP joints, and inadequate movement of the finger joints, as follows:

1. MPjoint extension alone will result in fixation in that position (collateral ligament shortening), with (a) pseudofiexion (dorsal interosseous palmar ligament insertions), (b) snap phenomenon (collateral ligament shortening), c) muscle wasting (disuse atrophy), and deformity (muscle dominance of volar interossei).

The lesion may apply to one finger only, but usually more than one are affected as the neighbouring joints in relation to the pathological one (septic arthritis, metacarpo-phalangeal fracture, single extensor tendon injury adhesions) also become affected. When the wrist can be extended, and the fingers are therefore strong and the thumb opposed, the disability applies only to the MP joints, but the wrist position is the key to the further and more severe state, when stiff fingers and adduction of the thumb are superadded.

2. Wrist drop produces the following developments:

(a) It allows extension of the MP joint through the pull on the extensor tendons and slack of the flexor tendons and lumbricals, and, even if there is no local pathology, fixation may occur through wrist drop alone, maintained through neglect or continued pain. This is seen also in many other lesions that are associated with wrist drop maintained for a long time. The position of ease is not the position of function. Any hand allowed to assume the position of ease, with a supported elbow, will fall into that dreadful position of wrist drop and extended MP joints. And how much worse it is if pain or cumbersome dressings restrict movements as well! (b) Power is lost in the fingers because the flexor tendons are slack. Weak movements at the IP joints without full range will lead to stiffness and eventually fixation of the finger joints.

(c) The thumb extends and adducts from extensor tendon pull and slackness of the short thumb muscles of the thenar eminence. When the long flexor of the thumb contracts and the IP joint of the thumb now flexes, the thumb is adducted further, with tightening of the long extensor of the thumb and 'bow-stringing' of the thumb further into adduction.

The picture of the fully developed fixed claw hand is thus one of fixed MP joints in extension, atrophy and deformity, wrist drop, weak, partially-fixed IP joints, and a fixed adducted thumb.

The anatomical explanation of the progression to fixed claw hand is the opposite of that involved in the process of recovery of a hand in the position of function. (See Fig. 5, A, B, C and D, and Fig. 6, A, B, C and D.)

\section{Treatment.}

MP joints in flexion never get stiff, and early recover movement. Extension of the wrist assists MP joints to flex, gives power to fingers, and allows the thumb to become opposed and to recover in that position.

In a bad hand, one must get the wrist up, and the IP joints moving. In the early stages of an impending claw hand, get the MP joints forward into flexion. It has been said that there is no treatment for a fixed claw hand. This is not true. Dorsal fibres of the MP collateral ligaments can be cut, or the collateral ligamants manipulated by flexing the MP joints, and the hand can be put up in a position of function. This must be maintained for months; and the patient will recover useful hand function.

In the worst hands, start with the MP joints maintained in flexion by the use of plaster of Paris; the IP joint can then use the plaster cross-slab as leverage, with or without elastic traction. The wrist is well extended to give full power to the IP joint movements through the flexor tendons. The thumb is opposed. This position may be maintained for weeks. In one case*, the plaster of Paris was left on for 2-3 months; full recovery took place.

Physiotherapists frown and plead for removal of the plaster of Paris. This is done when recovery is on its way. Wrist drop and mobility certainly aid recovery of extension and the return of MP movements, with the altering position of the wrist, but it is my experience that this will always come if the MP joints have been kept in flexion. They cannot get stiff in this position, unless skin scars or tendon scar to skin is present in the palm.

It is important to recognise the hand in early stages of fixation. If resistance to flexion is encountered at the MP joints, act at once, and manipulate the MP joints into flexion and maintain them so for about a week; the recovery will be dramatic.

\section{FINGER DEFORMITIES}

Finger Deformities from Hyperextension of the Proximal Interphalangeal joints with Flexion of the Distal joints.

This condition may occur from many causes. Kaplan has stated that if the capsule of the joint ruptures hyperextension occurs, but an analysis of cases shows the following:

(a) Physiological. Some people can fiex the distal IP joints while still maintaining the proximal IP joint extension. This is the reverse of normal, in which flexion occurs first in the proximal joint and the lateral bands 'button-hole' over the sides of the joint to give length to the extensor tendon for subsequent flexion of the distal joint. When the distal joint is flexed first, the lateral bands are tautened into extension, and 'bow-string' the proximal IP joint into hyperextension.

(b) Burns. In burns, scar under the distal joint will produce this deformity; so will scar over the proximal phalanx, which prevents the lateral bands 'button-holing', so that the distal joint starts to flex.

* Illustrated at the meetings by a diagram. 
(c) Dupunteris Confracuie. This deformity has also been seen in Dupuytren's contracture. What hatppens is that the proximal IP joint overcomes the scar on the volall surface. In the patient's efforts at cxtension of the fingers with the MP joints sull flexed by the deformity, the intrinsic muscles overact, and the lateral bands pass dorsally. In the continued maintained extension of the proximal IP joint. flexion of the distal IP joints further bow-strings the lateral bands dorsally - hence the deformily. (Sce Fig. 7.)

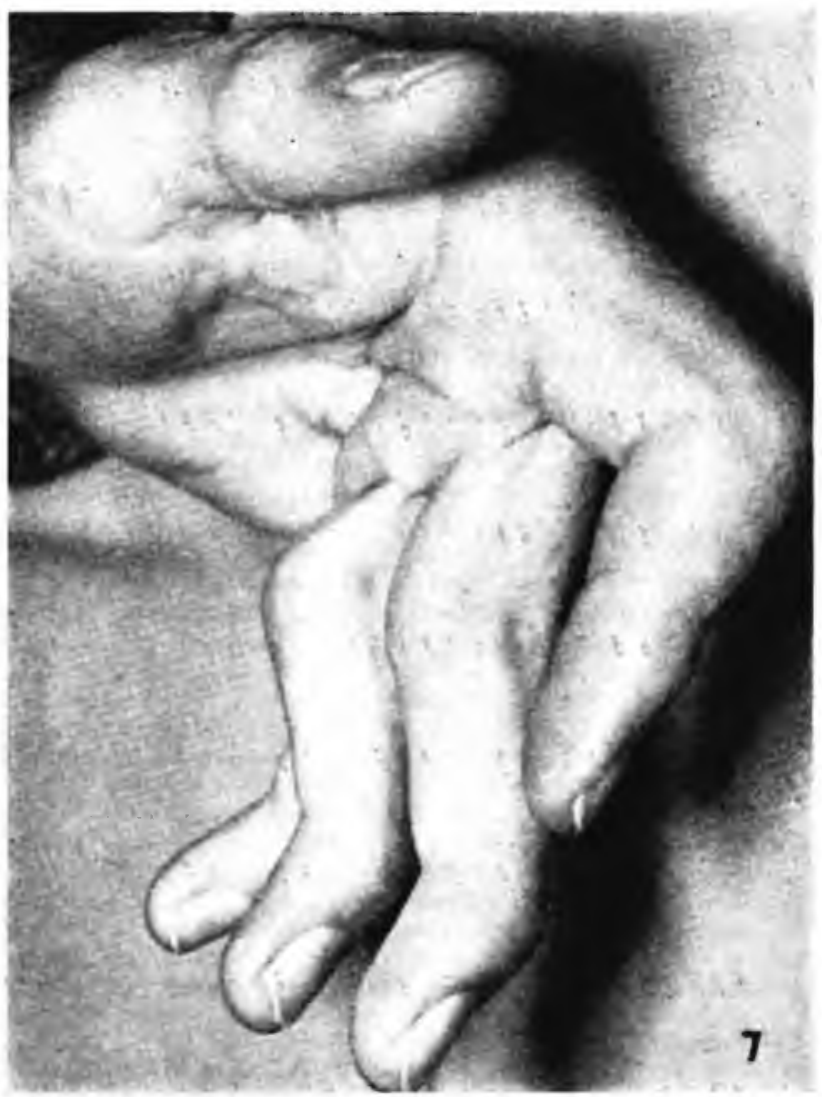

fie. 7. Dupusiren's contracture

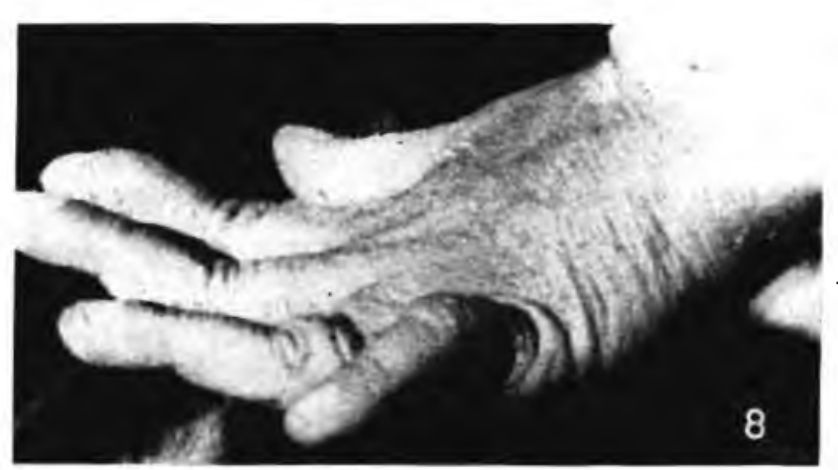

Fig. 8. Post-paralytic ispe of hyperextension deformils of prosimal IP joint. (d) Posi-paralyic. A similar deformily has been seen in a post-paralytic sasc. and here a weatkness of extensors and compensaltory over-function of the intrinsics seems to be the basis for the production of an "intrinsic plus" hand. It is reported that a similar deformity sometimes occurs when the profundus tendon flexes the distal IP joint in the absence of a sublimis to flex the proximal IP joint-but not in my experience. (See Fig. 8.)

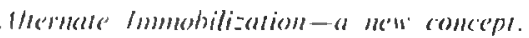

Bumbu-hole deformity is due to loss of funclion of the centril slip of the extensor cendon. The proximal IP joint flexes and the distal joint cxtends.

The repair of this deformity is surgical. and the results often poor. Simple suture is ineffective or else results in loss of function and stiftness in extension.

There are lwo funclional clements in the aponeurosis. viz. (1) the central extensor mechanism at the proxinal IP joint, and (2) the mechanism for the lateral band, which glides sepitrattely on movement of the distal IP joint. When an extensor tendon and aponeurosis are sutured, at first the proximal IP joint should be immobilised. while union occurs in the sutured central extensor tendon. This likes approximaltely 5 weeks, and one must allow freedom of mosement in the distal IP joint. to keep the gliding action of the latteral batnds. Subsequently, the distat joint should be immobilized and the proximal joint allowed free, so that the lateral bands can move over ihe sides in the true fashion, and fiexion and extension develop at the proximal IP joint. The centril extensor tendon develops its function of extending the proximal IP joint in a co-ordinatled fashion in relation (o) the distal joint.

Alternille finger joint immobilisation hals a plate in the treattment of any injury of the extensor aponeurosis.

Finger Sitfores. A case illustrating the cattuse of tinger stiffness. and the treatment, is worth mentioning. A young lady developed a glonius tumour on the pulp of her right index finger, and it wals so painful that affer tratment she was seen eventually with a complctely stifl finger in extension. There wals no other cause than inmobilizatlion.

A fill) wats turned al the proximat IP joint. and the lalleral and eentral slips of the aponeurosis separated at this point, exposing the dorsal tibres of the collateral ligaments, which were then separalsed and excised from the underlying head of the phalanx. Flexion becante possible all this stage, and recovery ensued with return of full funclion.

For recovery in tinger stiffness, the collatcral ligitments nust allow movement, either having been strelched or cut or assisted by elastic traction. A determined pitient is needed. able to co-operate in the face of pain: and thers musı be atl extended wrisı 10 give full power 10 flexion by fiexor icndons free of scar and in continuity, and extensor tendons. 10o. free of adhesions, 10 allow flexion to occur.

\section{MISCFLLANEOUS}

In two cases of tolally disabled hands from ciush injuries which hald resulted in melacarpal fracture with flexor and extensor tendon fixation to deep and skin sciar, and with wrist drop. MP extension and fixition, and thumb adduction, a considerable degree of recovery was possible by the following measures: (i) Frecing tendons, (ii) resecting portions of metalarpals where scar and deformity made recurrence of adhesions to lundons inevitable. (iii) flexing MP joints, (iv) fixaltion by platster of Paris wilh wrist extension, MP joint fexion ind crossbar leverage for fingers, (v) elastic traction, and (vi) clectric stimulation of flexors and extensors al an carly stage.

Tendon Traniylanes and Giafis

In litte poliomyclitis cases, tbe results of tendon transplants have been very salisfactory, combined sometimes with arthrodesis of the wrist

In spastic cases, operations may relieve deformity, but rarely gise much improvencent in function. 


\section{Skin Corcrage.}

Perhaps the most important advance in hand surgery in recent years is the use of skin coverage when needed. Time and again early skin placement over tendons. bone and exposed joints has salved limb and function.

One caln walit a weck 10 assess the degrec of necrosis in skin and tendon. etc., for it is not always possible inmediately to asscss the degree of damage, which is almost always more than the first impression suggesis. Even a longer lapse of time docs not prevent coverage. When one is in doubt in the early stitges, a dressing of tulle gras tied over with some pressure will almost always ensure at healthy surfice on inspection alt the end of the week.

In a case seen 18 dalys after gross injury. in which the dorsil structures of the right forearm. wrist and dorsum of the hand were lost. bone denuded of periosteum was visible including lengths of radius. ulna and metacarpals. Free pus escaped from the wrist areil. and the ring and small fingers and the metacarpals were loose and necrotic.

Carpal bones were excised. and the flexor tendons could be seen from the dorsum bathed in pus. Free drainage was established in the palm. and a dressing of tulle gras was sewn into the place with pressure

A weck later, al litrge abdominal flap wals used as coverage. The cxposed bones werc nibbled until bleeding occurred. Healing took place without sinuses and. within a short time. extensor tendon grifts to the abductors and extensors of the thumb and remaining fingers could be dons through the naps.
Onc learns from this casc thalt. alter 18 days of exposure of bone and tendons. coverage is still possible. and this has been our experience with other cases of exposed bones and joints in neglected compound fractures and dislocations of the ankle and tibia.

Different methods of reconstructing thumbs can be accomplished. such als by skin pedicle from the abdomen. and later bone grafting. Transplantation of a stump of an umputated middle finger and metacarpal to the thumb has been used successfully. and so has rotation of a metacarpal. Each case should be considered in its own merits: we are always assisted by a good patient. and the natural adaptability of remaining digits for pratetical use.

\section{SUMMNRY}

In this talk, an effort has been mide to explain the anatomical reasons for recovery of a correctly positioned hand as against the deterioration 10 a fixed claw hand in a poorly positioned hand.

The deformity of hyperextension of proxintal interphalangeal joints with flexion of distal interphalangeal joints has been shown to be due to several interesting mechanical causes.

The concept of alternatc immobilization to retain and restore finger moventents is propounded. Ealrly skin coverage by flaps of exposed tendons. bone and joints is strongly advocated at the monent the necrosis of covering skin is discernable.

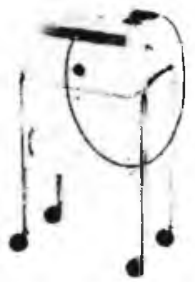

THE BIRTCHER MEGASON VI ULTRASONIC UNIT

To the more than 20,000 physicians now using ultrasonic therapy in the treatment of a host of acute and chronic conditions, this precision instrument adds new dimensions of accuracy and treatment ease.

Descriptives and medical journal reprints on request.
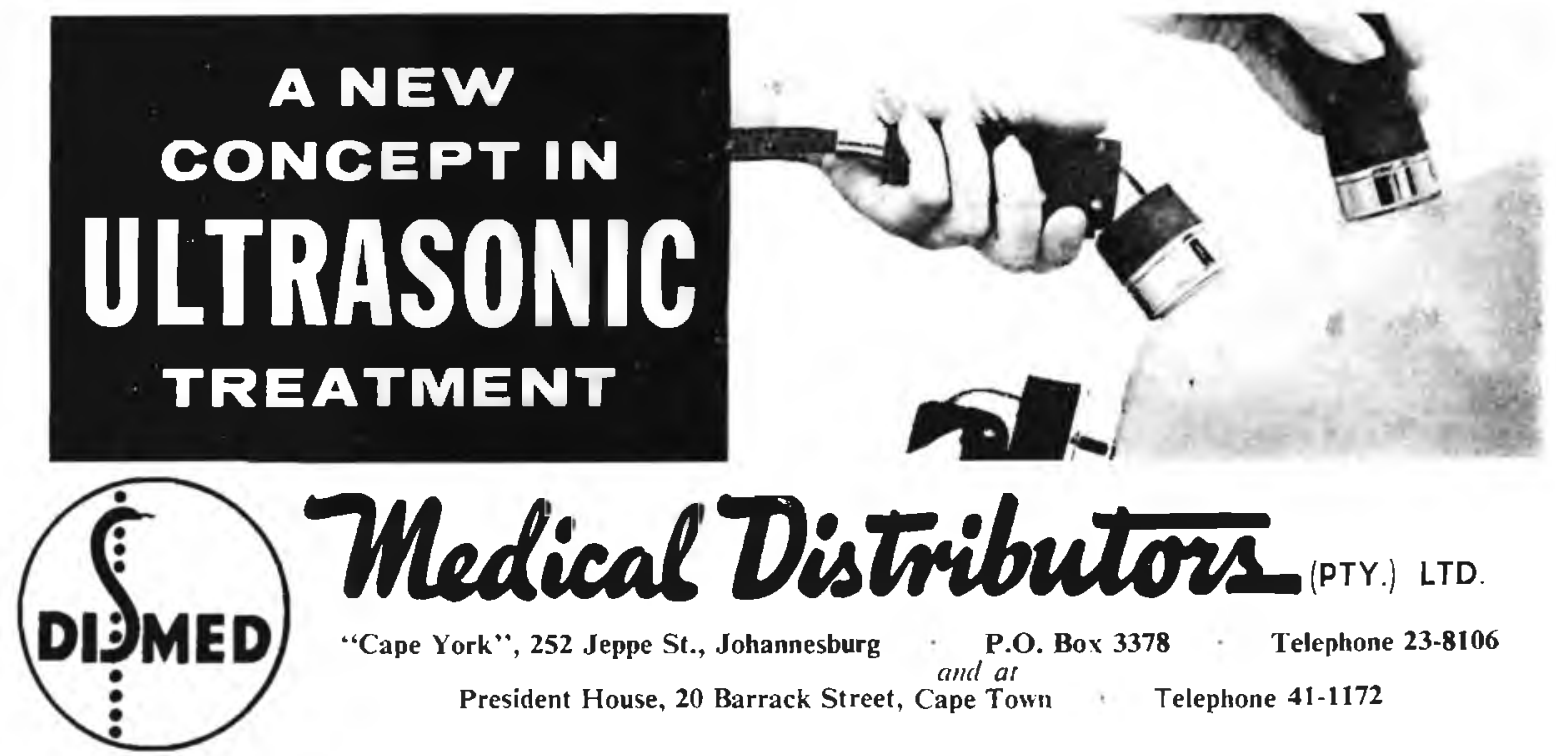

“Cape York", 252 Jeppe St., Johannesburg and a

P.O. Box 3378

Telephone 23-8106

President House, 20 Barrack Street, Cape Town

Telephone 41-1172 